\title{
Switching on magnetism in Ni-doped graphene: Density functional calculations
}

\author{
E. J. G. Santos, ${ }^{1,2, *}$ A. Ayuela, ${ }^{1,2, \dagger}$ S. B. Fagan, ${ }^{3}$ J. Mendes Filho, ${ }^{4}$ D. L. Azevedo, ${ }^{5}$ \\ A. G. Souza Filho, ${ }^{4}$ and D. Sánchez-Portal ${ }^{1,2,+}$ \\ ${ }^{1}$ Centro de Física de Materiales, Centro Mixto CSIC-UPV/EHU, Apdo. 1072, 20080 San Sebastián, Spain \\ ${ }^{2}$ Donostia International Physics Center (DIPC), Paseo Manuel de Lardizabal 4, 20018 San Sebastián, Spain \\ ${ }^{3}$ Área de Ciências Naturais e Tecnológicas, Centro Universitário Franciscano, Santa Maria 97010-032, RS, Brazil \\ ${ }^{4}$ Departamento de Física, Universidade Federal do Ceará, Fortaleza 60455-760, CE, Brazil \\ ${ }^{5}$ Universidade Federal do Maranhão, São Luis 65080-040, MA, Brazil
}

(Received 4 September 2008; published 19 November 2008)

\begin{abstract}
Magnetic properties of graphenic carbon nanostructures, which are relevant for future spintronic applications, depend crucially on doping and on the presence of defects. In this paper we study the magnetism of the recently detected substitutional $\mathrm{Ni}\left(\mathrm{Ni}_{\text {sub }}\right)$ impurities. $\mathrm{Ni}_{\text {sub }}$ defects are nonmagnetic in flat graphene and develop a nonzero-spin moment only in metallic nanotubes. This surprising behavior stems from the peculiar curvature dependence of the electronic structure of $\mathrm{Ni}_{\text {sub }}$. A similar magnetic-nonmagnetic transition of $\mathrm{Ni}_{\text {sub }}$ can be expected by applying anisotropic strain to a flat graphene layer.
\end{abstract}

DOI: 10.1103/PhysRevB.78.195420

PACS number(s): 75.75.+a, 68.43.Fg, 73.20.Hb, 73.22. -f

\section{INTRODUCTION}

Graphenic carbon nanostructures have opened new research routes in nanoelectronics. ${ }^{1,2}$ In particular, their magnetic properties are receiving much attention, both experimentally and theoretically, mainly in relation to spintronics. Spin qubits and other spintronic devices seem feasible due to the very long spin relaxation and decoherence times in graphene $e^{3,4}$ and to the fact that the magnetism of the edge states of graphenic nanoribbons can be controlled by applying external electric fields. ${ }^{5,6}$ Magneto-optical properties are also being actively studied. ${ }^{2,7}$ All these properties are drastically influenced by the presence of defects and dopants. ${ }^{8-10}$ For example, strong magnetic signals in nanocarbons have been reported after irradiation that seem to be associated with the creation of defects. ${ }^{11-14}$ Thus, the magnetic and transport properties of carbon systems can in principle be engineered using these additional degrees of freedom.

In a recent x-ray adsorption study, Ushiro et al. ${ }^{15}$ demonstrated the presence of important amounts of substitutional $\mathrm{Ni}\left(\mathrm{Ni}_{\text {sub }}\right)$ impurities in purified carbon nanostructures synthesized using Ni-containing catalyst. Such $\mathrm{Ni}_{\text {sub }}$ impurities were also observed by Banhart et al. ${ }^{16}$ in electron microscopy images of onionlike graphenic particles. In general, due to the stability of the substitutional configuration, the incorporation of transition metals to the carbon layer during growth or saturating existing vacancies seems as a likely process. In spite of this, the magnetic properties of substitutional transition-metal impurities in graphenic systems have not been studied in detail. Instead, most theoretical studies have focused on adsorbed transition-metal atoms on fullerenes and single-walled carbon nanotubes (SWCNTs). ${ }^{17-23}$ Few calculations to date have considered $\mathrm{Ni}_{\text {sub }}$ impurities ${ }^{16,24}$ and little attention was paid to the magnetic properties.

In this paper we present a first-principles density functional theory (DFT) study of $\mathrm{Ni}_{\text {sub }}$ defects in graphene and armchair and zigzag SWCNTs of different diameters. We have discovered that the spin moment of substitutionally $\mathrm{Ni}$ - doped graphene can be controlled by applying mechanical deformations that break the hexagonal symmetry of the layer, as curvature does. Surprisingly, $\mathrm{Ni}_{\text {sub }}$ impurities are nonmagnetic in flat graphene. However, their spin moment can be switched on by applying curvature to the structure. Furthermore, the spin moment of $\mathrm{Ni}_{\text {sub }}$ also becomes a signature of the metallicity of the structure. Only metallic tubes develop a moment that depends on the tube diameter and Ni concentration. This behavior stems from the peculiar curvature dependence of the electronic structure of the $\mathrm{Ni}_{\text {sub }}$ impurity.

\section{METHODOLOGY}

Our calculations have been performed with the SIESTA code $^{25-28}$ using the generalized gradient approximation (GGA) (Ref. 29) to DFT and Troullier-Martins ${ }^{30}$ pseudopotentials. Our Ni pseudopotential includes a pseudocore with a core matching radius of 0.53 a.u. in order to perform nonlinear core corrections for the description of exchange and correlation. ${ }^{31}$ We have tested that this pseudopotential yields the correct magnetic moment and band structure for bulk Ni. We have used an energy shift ${ }^{26}$ of $50 \mathrm{meV}$ to define the radii of the different orbitals. A double- $\zeta$ (DZ) polarized (DZP) basis set has been used for the calculation of the spin moments and electronic band structures of all our systems. However, we have checked that a DZ basis yields to almost identical relaxed structures as the DZP basis and, therefore, we have used the smaller DZ basis for the relaxations of systems containing more than $\sim 100$ atoms (our supercells contain up to 512 atoms; see Fig. 5 below). Atomic coordinates were relaxed using a conjugated gradient algorithm until all force components were smaller than $0.05 \mathrm{eV} / \AA$. Relevant lattice parameters were fixed to those of the pristine systems. To prevent spurious interactions the minimum distance between the walls of neighboring SWCNTs was $18 \AA$. The fineness of the real-space grid used to calculate the Hartree and exchange-correlation contribution to the total energy and the Hamiltonian was equivalent to a 180 Ry plane-wave cutoff. A good integration over the Brillouin zone proved to 
(a)

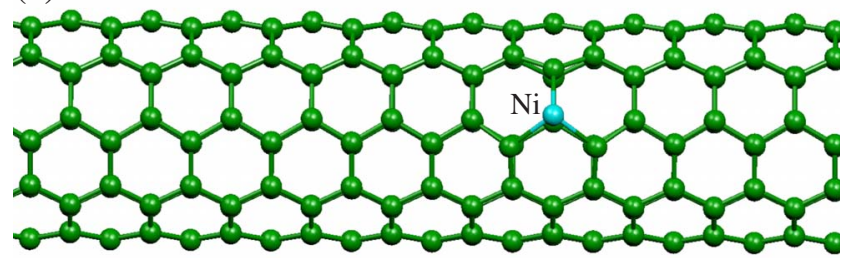

(b)

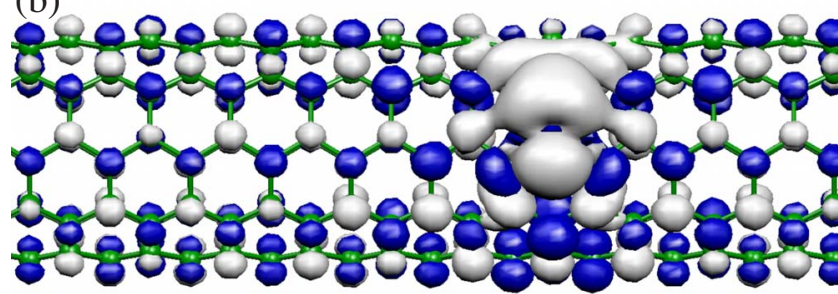

FIG. 1. (Color online) (a) Relaxed geometry of a $\mathrm{Ni}_{\text {sub }}$ impurity in a $(5,5)$ SWCNT and (b) isosurface $\left( \pm 0.004 e^{-} /\right.$bohr $\left.^{3}\right)$ of the magnetization density with light (gray) and dark (blue) surfaces corresponding, respectively, to majority and minority spins.

be instrumental to converge the spin moment of $\mathrm{Ni}_{\text {sub }}$ impurities in metallic SWCNTs. The $k$-point sampling was equivalent to a $136 \times 136$ sampling $^{32}$ of the Brillouin zone of graphene. We used a Fermi-Dirac distribution with $k_{B} T$ $=21 \mathrm{meV}$. In some cases we have checked that our results are reproduced using a different methodology: the VASP code with projected-augmented-wave potentials and a plane-wave cutoff energy of $400 \mathrm{eV}^{33,34}$

\section{RESULTS AND DISCUSSION}

Figure 1(a) illustrates the typical equilibrium structure of $\mathrm{Ni}_{\text {sub }}$ in the case of a $(5,5)$ SWCNT. The Ni atom appears displaced $\sim 0.9 \AA$ from the carbon plane. Although both outward and inward displacements can be stabilized, the outward configuration is always more stable. The calculated $\mathrm{Ni}-\mathrm{C}$ distances $\left(\mathrm{d}_{\mathrm{NiC}}\right)$ are in the range of $1.77-1.85 \AA$ in agreement with experiment. ${ }^{15,16}$ Armchair tubes exhibit two slightly shorter and one larger values of $\mathrm{d}_{\mathrm{NiC}}$. The opposite happens for $(n, 0)$ tubes, whereas for graphene we obtain a threefold symmetric structure with $\mathrm{d}_{\mathrm{NiC}}=1.78 \AA$. Ni adsorp- tion inhibits the reconstruction ${ }^{35}$ of the carbon vacancy. Furthermore, we have checked that a symmetric structure is obtained even when starting from a relaxed vacancy. The $\mathrm{Ni}$ binding energy is quite large: $7.9 \mathrm{eV}$ for graphene and about $8.5 \mathrm{eV}$ for $(5,5)$ and $(8,0)$ tubes. The calculated adsorption energy of $\mathrm{Ni}$ on the surface of the same tubes is $\sim 2.5 \mathrm{eV} .^{21}$ Thus, we can conclude that the formation of $\mathrm{Ni}_{\text {sub }}$ defects by passivation of existing carbon vacancies is a very likely process both for graphene and for SWCNTs.

Figure 1(b) shows the magnetization density for a $\mathrm{Ni}_{\text {sub }}$ defect in a $(5,5)$ metallic nanotube at large dilution $(0.3 \% \mathrm{Ni}$ concentration). The total spin moment of this system is $0.5 \mu_{B}$. The magnetization comprises the $\mathrm{Ni}$ atom and its $\mathrm{C}$ neighbors. However, it also extends considerably along the tube, particularly in the direction perpendicular to the tube axis. This indicates the polarization of some of the delocalized electronic states in the nanotube. Indeed, as we clarify below, the magnetism in substitutionally Ni-doped SWCNTs only appears associated with the curvature and the metallicity of the host structure.

Figure 2(a) shows a scheme of the electronic structure of $\mathrm{Ni}_{\text {sub }}$ in graphene, while Fig. 2(b) presents the calculated band structure using a $4 \times 4$ supercell. Very similar results are obtained using larger supercells. ${ }^{36}$ Several levels with $\mathrm{Ni}-\mathrm{C}$ bonding character and a strong $\mathrm{Ni} 3 d$ contribution can be found between 2 and $6 \mathrm{eV}$ below the Fermi energy $\left(E_{F}\right)$. This considerable bandwidth is a signature of the strong $\mathrm{Ni}-\mathrm{C}$ interaction. As a consequence of the bonding interaction the Ni $3 d$ band is stabilized and can be pictured as "fully occupied." Close to $E_{F}$ we find three levels with Ni-C antibonding character. One of them is occupied and appears around $0.7 \mathrm{eV}$ below $E_{F}$ close to $\Gamma$. This level comes from a fully symmetric linear combination of the $2 p_{z}$ orbitals $(z$-axis normal to the layer) of the nearest $\mathrm{C}$ neighbors interacting with the $3 d_{z^{2}}$ orbital of Ni. Two levels coming from the hybridization of the in-plane $s p$ lobes of the carbon neighbors with the Ni $3 d_{x z}$ and Ni $3 d_{y z}$ orbitals appear $\sim 0.5 \mathrm{eV}$ above $E_{F}$.

As a consequence of this electronic structure, with the Ni $3 d$ states well below $E_{F}$ and no flat bands crossing $E_{F}$, the spin moment of the $\mathrm{Ni}_{\text {sub }}$ impurity in graphene is zero. Interestingly, the three levels appearing close to $E_{F}$ in Fig. 2 are reminiscent of those found for the unreconstructed carbon
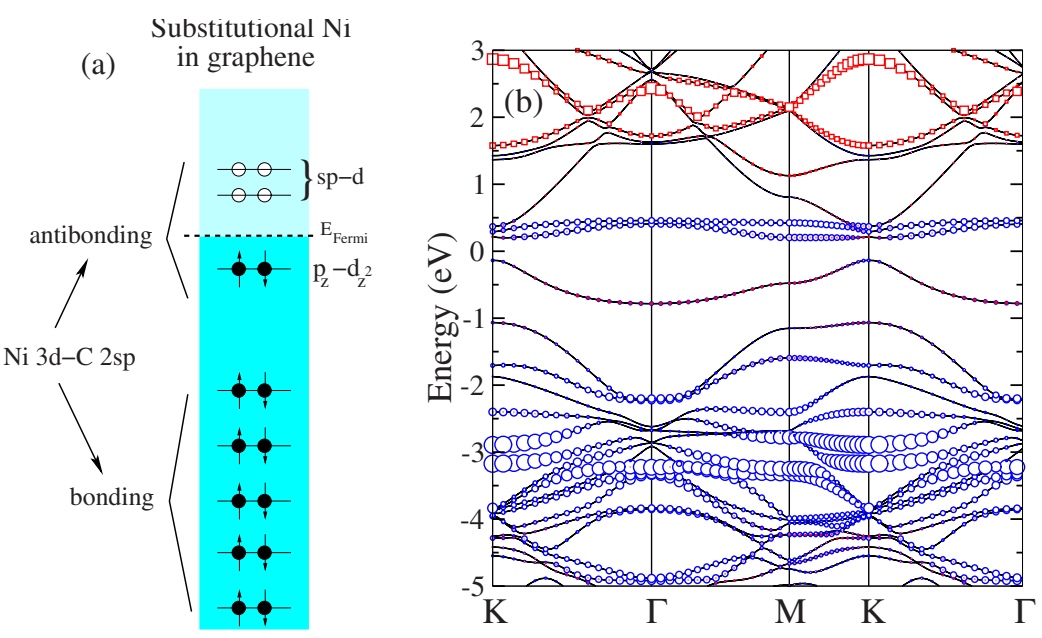

FIG. 2. (Color online) (a) Schematic representation of the electronic structure of a $\mathrm{Ni}_{\text {sub }}$ impurity in graphene. Panel (b) shows the calculated band structure for a $\mathrm{Ni}_{\text {sub }}$ impurity in a $4 \times 4$ graphene supercell. The size of the circles and squares corresponds to the amount of $\mathrm{Ni} 3 d$ and $\mathrm{Ni} 4 s$ characters, respectively. Energies are referred to the Fermi energy. 

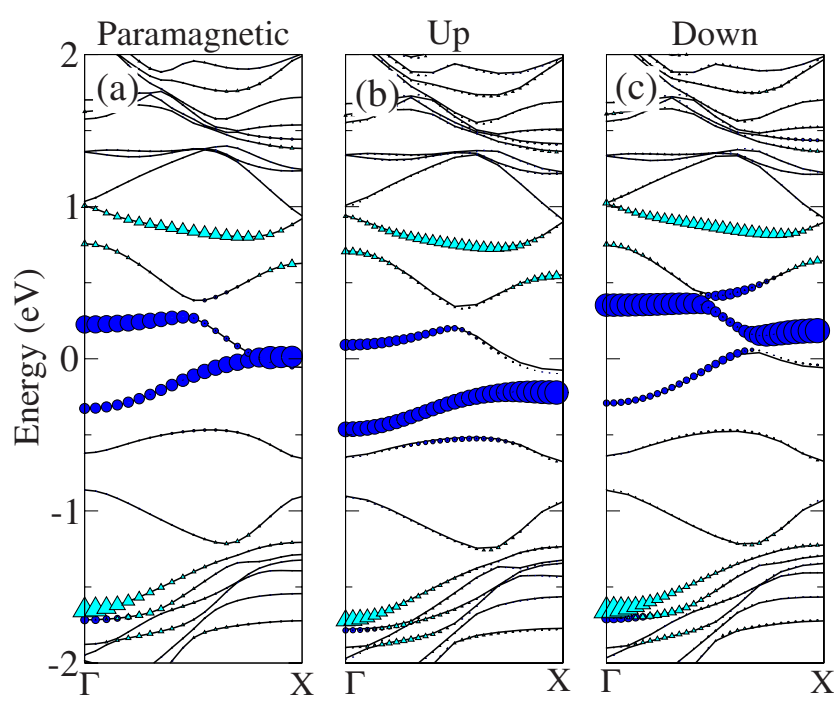

FIG. 3. (Color online) Band structure of a $(5,5)$ nanotube containing a $\mathrm{Ni}$ impurity every four unit cells $\left(\mathrm{Ni}_{\text {sub }}-\mathrm{Ni}_{\text {sub }}\right.$ distance of $\sim 9.8 \AA$ ) for (a) a spin-compensated calculation and for (b) majority and (c) minority spins. Circles and triangles correspond, respectively, to the amount of $\mathrm{Ni} 3 d_{y z}$ and $\mathrm{Ni} 3 d_{x z}$ characters. $x$ axis is parallel to the tube axis and $y$ axis is tangential.

vacancy in graphene. ${ }^{35}$ The presence of a Ni atom stabilizes such symmetric structure of the vacancy and slightly shifts the two levels coming from the $s p$ lobes (now C $2 s p-N i 3 d$ antibonding levels) to higher energies. This contributes to stabilize the paramagnetic solution and renders a $\mathrm{Ni}_{\text {sub }}$ impurity with zero-spin polarization in the flat layer of graphene.

The basic picture described above is still valid for the electronic structure of the $\mathrm{Ni}_{\text {sub }}$ impurity in SWCNTs. However, the modifications that appear due to the curvature of the carbon layer are responsible for the appearance of a spin moment. Figure 3(a) shows the band structure of a paramagnetic calculation of a $(5,5) \mathrm{SWCNT}$ with a $\mathrm{Ni}_{\text {sub }}$ impurity every four unit cells. Here the distance between neighboring $\mathrm{Ni}_{\text {sub }}$ impurities is similar to that of the graphene layer in Fig. 2(b), although the $\mathrm{Ni}$ concentration is 2.5 times lower $(1.3 \%)$. Comparing these two figures we can appreciate the effects of curvature. The degeneracy between $d_{x z}$ and $d_{y z}$ states is removed ( $x$ axis taken along the tube axis and $y$ axis along the tangential direction at the $\mathrm{Ni}$ site). The $d_{y z}$ contribution is stabilized by several tenths of electron volt and a quite flat band with strong $d_{y z}$ character is found pinned at $E_{F}$ close to the Brillouin-zone boundary. Under these conditions the spin-compensated solution becomes unstable and a spin moment of $0.48 \mu_{B}$ is developed. Figures 3(b) and 3(c) show, respectively, the band structure for majority and minority spins. The exchange splitting of the $d_{y z}$ level is $\sim 0.4 \mathrm{eV}$ and the energy gain with respect to the paramagnetic solution is $32 \mathrm{meV}$. Similar results are obtained using the VASP code. Notice that this exchange splitting is much smaller than the typical values obtained for transition metals. This is a consequence of the quite delocalized character ${ }^{36}$ of the impurity level responsible for the spin polarization and translates into the sensitivity of the $\mathrm{Ni}_{\text {sub }}$ spin moment to relatively small perturbations. This is reflected, for example, in the complex dependence (see below) of the spin moment on the particular
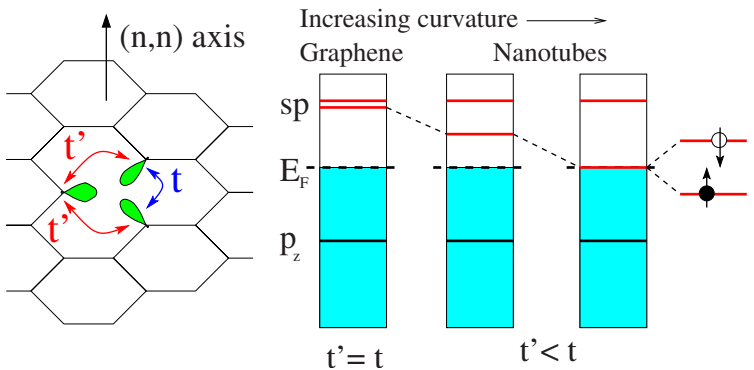

FIG. 4. (Color online) Effect of curvature (anisotropic strain) on $\mathrm{Ni}_{\text {sub }}$ in $(n, n)$ carbon nanotubes. The electronic structure of $\mathrm{Ni}_{\text {sub }}$ close to the Fermi level is similar to that of the unreconstructed carbon vacancy. One of the impurity levels with antibonding C $2 s p$-Ni $3 d$ character is shifted downward and, for large enough curvatures, becomes partially populated and spin polarized.

arrangement of $\mathrm{Ni}_{\text {sub }}$ defects in the tube and the tube diameter.

In general, whenever a flat impurity with appreciable Ni $3 d$ character becomes partially filled we can expect the appearance of a spin moment. The population of such an impurity level occurs at the expense of the simultaneous depopulation of some of the delocalized carbon $p_{z}$ levels of the host structure. For this reason the development of a spin moment is more likely for $\mathrm{Ni}_{\text {sub }}$ impurities in metallic structures such as the armchair tubes. The crucial role of the host states also explains the delocalized character of the magnetization density depicted in Fig. 1(b). However, it is important to stress that the driving force for the formation of a spin moment associated with the $\mathrm{Ni}_{\text {sub }}$ impurity in SWCNTs is the local curvature of the carbon layer that shifts the energy position of one of the impurity levels downward until it crosses $E_{F}$. A schematic representation of this phenomenon can be found in Fig. 4 where we also emphasize the similarities between the levels of the $\mathrm{Ni}_{\text {sub }}$ defect and those of the unreconstructed carbon vacancy. At large tube diameters we must recover the limit of flat graphene with zero magnetic moment.

For semiconducting tubes the situation is somewhat different. The $d_{x z}$ and $d_{y z}$ derived levels will remain unoccupied unless their energies are shifted by a larger amount that pushes one of them below the top of the valence band. Therefore, if the tube has a large enough gap the spin moment will be zero irrespective of the tube diameter. We have explicitly checked that a zero-spin moment is obtained for $(8,0)$ and $(10,0)$ semiconducting tubes for Ni concentrations ranging from $1.5 \%$ to $0.5 \%$. The different magnetic behavior of $\mathrm{Ni}_{\text {sub }}$ impurities depending on the metallic and semiconducting characters of the host structure provides a route to experimentally identify metallic armchair tubes.

Figure 5 displays the spin moment per $\mathrm{Ni}_{\text {sub }}$ atom for Ni-doped armchair tubes of different diameters. All of them present a spin moment that oscillates as a function of the tube diameter and the size of the supercell used in the calculation, i.e., the $\mathrm{Ni}_{\text {sub }}-\mathrm{Ni}_{\text {sub }}$ distance. For $(4,4),(5,5)$, and $(6,6)$ tubes the first supercell showing a nonzero-spin moment contains four unit cells. For $(7,7)$ and $(8,8)$ tubes this minimum length increases up to eight and six unit cells, respectively. The complex oscillatory pattern of the spin moment as 


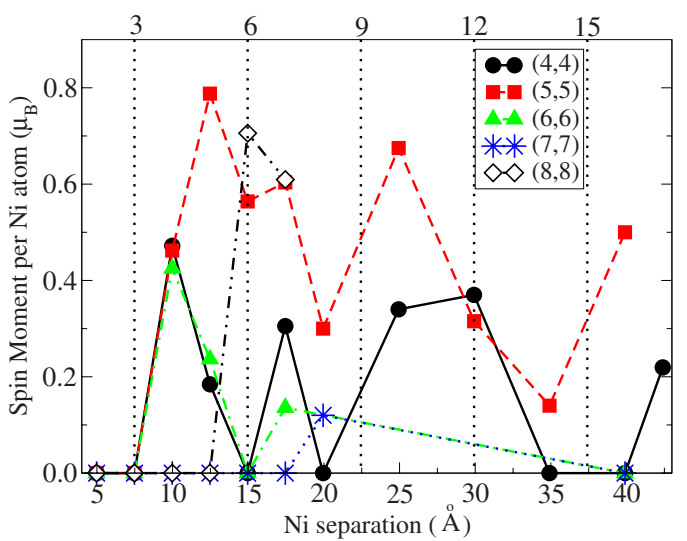

FIG. 5. (Color online) Spin moment per Ni impurity for different $(n, n)$ tubes as a function of the distance between periodic images of the impurity, i.e., the length of the supercell. The vertical lines and the numbers at the top indicate the number of unit cells in a supercell of a given length.

a function of the $\mathrm{Ni}_{\text {sub }}-\mathrm{Ni}_{\text {sub }}$ distance can be understood if we recall that the appearance of spin polarization critically depends on the energy position of a particular impurity level (C $2 s p-N i 3 d_{y z}$ ) nearby $E_{F}$. This impurity level has a relatively delocalized character and presents a strong hybridization with the electronic states of the nanotube. ${ }^{36}$ This translates into a long-range effective interaction between impurity states in neighboring $\mathrm{Ni}_{\text {sub }}$ defects. As a consequence, the exact position of the impurity level in each defect, and thus the possibility to develop spin polarization, depends on the particular arrangement of neighboring $\mathrm{Ni}_{\text {sub }}$ impurities. This gives rise to strong oscillations of the spin moment even for very large $\mathrm{Ni}_{\text {sub }}-\mathrm{Ni}_{\text {sub }}$ distances [see, for example, the $\mathrm{Ni}_{\text {sub }}$ spin moment in $(5,5)$ and $(4,4)$ tubes for $\mathrm{Ni}_{\text {sub }}-\mathrm{Ni}_{\text {sub }}$ distances between 20 and $40 \AA$ ]. Unfortunately, a meaningful exploration of larger distances between impurities requires a methodology different from the $a b$ initio supercell approach used here.

In Fig. 5 we have only considered systems with a single $\mathrm{Ni}_{\text {sub }}$ impurity per unit cell. Therefore, we are restricted to consider the ferromagnetic (FM) order of the spin moments associated with the $\mathrm{Ni}_{\text {sub }}$ defects. Unfortunately, in most cases the large size of the calculations prevents the doubling of the cell required to check different magnetic orders. However, we have explored also the possibility of antiferromagnetic (AFM) order in one case. We have used a $20 \AA$ supercell of a $(5,5)$ tube with two $\mathrm{Ni}_{\text {sub }}$ impurities and calculated FM and AFM arrangements. In this case the calculated spin moment in each $\mathrm{Ni}_{\text {sub }}$ was not only dependent on the relative positions of the $\mathrm{Ni}_{\text {sub }}$ impurities, but also showed a weaker dependence on the particular magnetic order (FM or AFM) considered. The FM configuration was always found to be the most stable except for situations where the two $\mathrm{Ni}_{\text {sub }}$ impurities shared common $\mathrm{C}$ neighbors. Only in those cases the spin-compensated solution became the most favorable.

Although more work is necessary to accurately determine the size and distance dependences of the effective exchange interaction (probably using model calculations similar to those in Ref. 37), our results indicate that $\mathrm{Ni}_{\text {sub }}$ impurities in small diameter metallic nanotubes, where they develop a spin moment, can exhibit relatively large FM couplings that slowly decay with distance. For example, in a $(5,5)$ tube the FM ordering of a collection of 10 - $\AA$-equispaced $\mathrm{Ni}_{\text {sub }}$ impurities along the tube axis is favored over the AFM configuration by as much as $36 \mathrm{meV}$ per $\mathrm{Ni}$ atom. These FM interactions can have important implications for the experimental detection of the curvature-dependent magnetism of the $\mathrm{Ni}_{\text {sub }}$ impurities described in this work and its influence in the observation of magnetism in carbon nanotube samples.

\section{CONCLUSIONS}

The calculations presented here show that substitutional $\mathrm{Ni}$ impurities in graphenic carbon structures present a strong covalent interaction with their carbon neighbors. This interaction stabilizes the $3 d$ levels of $\mathrm{Ni}$ that appear as a completely filled shell. As a consequence, $\mathrm{Ni}_{\text {sub }}$ is nonmagnetic in graphene. Two unoccupied levels of the $\mathrm{Ni}_{\text {sub }}$ impurity appear close to $E_{F}$ corresponding to antibonding combinations of $\mathrm{Ni} 3 d$ orbitals and $\mathrm{C} 2 s p$ lobes. Curvature breaks the degeneracy of these two levels and, under appropriate conditions, shifts one of them to lower energies such that it becomes partially occupied in metallic armchair tubes. In this situation the system develops a spin moment that exhibits a complex behavior as a function of the tube diameter and $\mathrm{Ni}$ concentration. However, for semiconducting zigzag tubes the $\mathrm{Ni}_{\text {sub }}$ impurities remain nonmagnetic. These results should be taken into account when studying the magnetic properties of carbon nanostructures.

In summary, curvature can be used to drive substitutional $\mathrm{Ni}$ impurities between a magnetic and a nonmagnetic state in metallic graphenic carbon nanostructures. In particular, the magnetic properties of substitutionally Ni-doped graphene can be tuned by controlling the curvature of the layer around the $\mathrm{Ni}_{\text {sub }}$ defects. A possible way to do this is to deposit the graphene layer on a substrate with a small lattice-parameter mismatch. This can cause the undulation and local bending of the graphene layer. ${ }^{38,39}$ Alternatively, a similar nonmagnetic-magnetic transition of $\mathrm{Ni}_{\text {sub }}$ might be obtained by applying anisotropic strain to a flat graphene layer. In this regard, we are currently exploring if the formation of a spin moment can also be controlled by applying uniaxial strain to a flat $\mathrm{Ni}_{\text {sub }}$-doped graphene layer.

\section{ACKNOWLEDGMENTS}

E.J.G.S. wants to thank S. Riikonen for useful discussions along this project. E.J.G.S., A.A., and D.S.P. acknowledge support from the Basque Government and UPV/EHU (Grant No. IT-366-07), CSIC, the Spanish MEC (Grant No. FIS2007-66711-C02-02), and the Basque Government and Diputación Foral de Guipuzcoa through the ETORTEK program. A.G.S.F. acknowledges the support from Brazilian agencies FUNCAP, CAPES/FAPERGS, CNPq (Grant No. 306335/2007-7), Rede Nacional de Pesquisa em Nanotubos de Carbono, Rede de Nanobioestruturas, and Instituto do Milenio de Nanotecnologia (CNPq/MCT-Brazil). 
*eltonjose_gomes@ehu.es

†swxayfea@ehu.es

*sqbsapod@ehu.es

${ }^{1}$ A. K. Geim and K. S. Novoselov, Nature Mater. 6, 183 (2007).

${ }^{2}$ Carbon Nanotubes: Advanced Topics in the Synthesis, Structure, Properties and Applications, edited by A. Jorio, M. S. Dresselhaus, and G. Dresselhaus (Springer-Verlag, Berlin, 2008).

${ }^{3}$ L. E. Hueso, J. M. Pruneda, V. Ferrari, G. Burnell, J. P. ValdésHerrera, B. D. Simons, P. B. Littlewood, E. Artacho, A. Fert, and N. D. Mathur, Nature (London) 445, 410 (2007).

${ }^{4}$ B. Trauzettel, D. V. Bulaev, D. Loss, and G. Burkard, Nat. Phys. 3, 192 (2007).

${ }^{5}$ Y.-W. Son, M. L. Cohen, and S. G. Louie, Nature (London) 444, 347 (2006).

${ }^{6}$ O. V. Yazyev and M. I. Katsnelson, Phys. Rev. Lett. 100, 047209 (2008).

${ }^{7}$ S. Zaric, G. N. Ostojic, J. Shaver, J. Kono, O. Portugall, P. H. Frings, G. L. Rikken, M. Furis, S. A. Crooker, X. Wei, V. C. Moore, R. H. Hauge, and R. E. Smalley, Phys. Rev. Lett. 96, 016406 (2006).

${ }^{8}$ B. Uchoa, V. N. Kotov, N. M. R. Peres, and A. H. Castro Neto, Phys. Rev. Lett. 101, 026805 (2008).

${ }^{9}$ L. Brey, H. A. Fertig, and S. Das Sarma, Phys. Rev. Lett. 99, 116802 (2007).

${ }^{10}$ I. Zanella, S. Guerini, S. B. Fagan, J. Mendes Filho, and A. G. Souza Filho, Phys. Rev. B 77, 073404 (2008).

${ }^{11}$ P. O. Lehtinen, A. S. Foster, A. Ayuela, A. V. Krasheninnikov, K. Nordlund, and R. M. Nieminen, Phys. Rev. Lett. 91, 017202 (2003).

${ }^{12}$ P. Esquinazi, D. Spemann, R. Höhne, A. Setzer, K.-H. Han, and T. Butz, Phys. Rev. Lett. 91, 227201 (2003).

${ }^{13}$ P. O. Lehtinen, A. S. Foster, Y. Ma, A. V. Krasheninnikov, and R. M. Nieminen, Phys. Rev. Lett. 93, 187202 (2004).

${ }^{14}$ A. V. Krasheninnikov and F. Banhart, Nature Mater. 6, 723 (2007).

${ }^{15}$ M. Ushiro, K. Uno, T. Fujikawa, Y. Sato, K. Tohji, F. Watari, W. J. Chun, Y. Koike, and K. Asakura, Phys. Rev. B 73, 144103 (2006).

${ }^{16}$ F. Banhart, J. C. Charlier, and P. M. Ajayan, Phys. Rev. Lett. 84, $686(2000)$

${ }^{17}$ Y.-H. Lee, S. G. Kim, and D. Tománek, Phys. Rev. Lett. 78, 2393 (1997).

${ }^{18}$ A. Ayuela, G. Seifert, and R. Schmidt, Z. Phys. D: At., Mol. Clusters 41, 69 (1997).

${ }^{19}$ G. Seifert, A. Bartl, L. Dunsch, A. Ayuela, and A. Rockenbauer, Appl. Phys. A: Mater. Sci. Process. 66, 265 (1998).

${ }^{20}$ M. Menon, A. N. Andriotis, and G. E. Froudakis, Chem. Phys. Lett. 320, 425 (2000).
${ }^{21}$ E. Durgun, S. Dag, V. M. K. Bagci, O. Gülseren, T. Yildirim, and S. Ciraci, Phys. Rev. B 67, 201401(R) (2003).

${ }^{22}$ Y. Yagi, T. M. Briere, M. H. F. Sluiter, V. Kumar, A. A. Farajian, and Y. Kawazoe, Phys. Rev. B 69, 075414 (2004).

${ }^{23}$ A. Grüneis and D. V. Vyalikh, Phys. Rev. B 77, 193401 (2008).

${ }^{24}$ A. N. Andriotis, M. Menon, and G. E. Froudakis, Phys. Rev. Lett. 85, 3193 (2000).

${ }^{25}$ D. Sánchez-Portal, P. O. E. Artacho, and J. M. Soler, Int. J. Quantum Chem. 65, 453 (1997).

${ }^{26}$ J. M. Soler, E. Artacho, J. D. Gale, A. García, J. Junquera, P. Ordejón, and D. Sánchez-Portal, J. Phys.: Condens. Matter 14, 2745 (2002).

${ }^{27}$ D. Sánchez-Portal, P. Ordejón, and E. Canadell, Struct. Bonding (Berlin) 113, 103 (2004).

${ }^{28}$ E. Artacho, E. Anglada, O. Diéguez, J. D. Gale, A. García, J. Junquera, R. M. Martin, P. Ordejón, J. M. Pruneda, D. SánchezPortal, and J. M. Soler, J. Phys.: Condens. Matter 20, 064208 (2008).

${ }^{29}$ J. P. Perdew, K. Burke, and M. Ernzerhof, Phys. Rev. Lett. 77, 3865 (1996).

${ }^{30}$ N. Troullier and J. L. Martins, Phys. Rev. B 43, 1993 (1991).

${ }^{31}$ S. G. Louie, S. Froyen, and M. L. Cohen, Phys. Rev. B 26, 1738 (1982).

${ }^{32}$ H. J. Monkhorst and J. D. Pack, Phys. Rev. B 13, 5188 (1976).

${ }^{33}$ G. Kresse and J. Hafner, Phys. Rev. B 47, 558 (1993).

${ }^{34}$ G. Kresse and D. Joubert, Phys. Rev. B 59, 1758 (1999).

${ }^{35}$ H. Amara, S. Latil, V. Meunier, P. Lambin, and J.-C. Charlier, Phys. Rev. B 76, 115423 (2007).

${ }^{36}$ Because of the quite symmetric position of the $\mathrm{Ni}$ atom over the $\mathrm{C}$ vacancy the $3 d_{x z}$ and $3 d_{y z}$ derived bands are almost degenerate at $\Gamma$. In spite of the relatively large distance between $\mathrm{Ni}$ atoms in the $4 \times 4$ graphene supercell $(\sim 9.8 \AA)$, these bands show a considerable dispersion of $\sim 0.2 \mathrm{eV}$. The large range of the $\mathrm{Ni}_{\text {sub }}-\mathrm{Ni}_{\text {sub }}$ interaction is a signature of the strong hybridization of the impurity levels with the states of the carbon layer. Another signature of this hybridization is the gap of $\sim 0.5 \mathrm{eV}$ that opens in the graphene layer around $\mathrm{K}$. Using a larger 8 $\times 8$ supercell i.e., increasing the $\mathrm{Ni}_{\text {sub }}-\mathrm{Ni}_{\text {sub }}$ distance to $\sim 19.7 \AA$, the width of the impurity bands and the gap decrease, respectively, to $\sim 20$ and $\sim 100 \mathrm{meV}$. The positions of the impurity bands are, however, very similar to the case of the smaller cell.

${ }^{37}$ D. F. Kirwan, C. G. Rocha, A. T. Costa, and M. S. Ferreira, Phys. Rev. B 77, 085432 (2008).

${ }^{38}$ A. T. NDiaye, S. Bleikamp, P. J. Feibelman, and T. Michely, Phys. Rev. Lett. 97, 215501 (2006).

${ }^{39}$ P. J. Feibelman, Phys. Rev. B 77, 165419 (2008). 